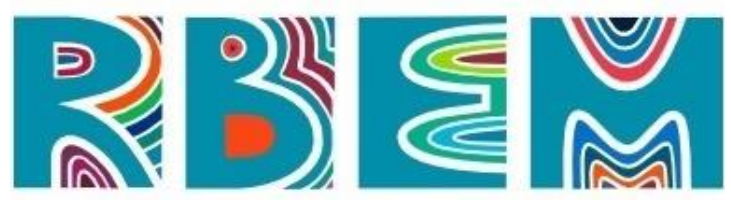

REVISTA BAIANA DE EDUCAÇÃO MATEMÁtICA

ARTIGO

dol https://doi.org/10.47207/rbem.v2i01.11286

\title{
Noção intuitiva no ato de ensinar e aprender matemática por meio de uma atividade de ensino de sistemas lineares com coeficientes positivos
}

\author{
PONTES, Edel Alexandre Silva \\ Professor Titular do Instituto Federal de Alagoas, Doutor em Ciências da Educação com ênfase no Ensino de \\ Matemática pela Universidad Tecnológica Intercontinental. ORCID. https://orcid.org/0000-0002-9782-8458 \\ E-mail: edel.pontes@ifal.edu.br
}

\begin{abstract}
Resumo: As dificuldades na compreensão de conceitos matemáticos na educação básica são evidentes no desempenho escolar dos educandos, especialmente pelas propostas educacionais suplantadas e que não conseguem aproximar nenhum interesse do aprendiz pelos conteúdos recomendados. Este trabalho tem como objetivo afrontar essas indagações e inquietudes de pesquisadores da Educação Matemática, estabelecendo três pressupostos indispensáveis para minimizar questionamentos sobre o ensino e aprendizagem de matemática: Problema, Causa e Paradigma. Metodologicamente, a proposta fundamenta-se em oferecer uma ferramenta intuitiva para resolução de sistemas lineares do $1^{\circ}$ grau com duas equações e duas incógnitas e coeficiente positivos, por intermédio de problemas matemáticos, utilizando uma tabela de dados. Quatro problemas matemáticos serão investigados. Não basta exclusivamente resolver o problema matemático indicado, mas, descobrir um caminho para desvendar padrões e estabelecer relações. Espera-se que este trabalho possa auxiliar professores e alunos no processo de ensino e aprendizagem de matemática, utilizando uma estratégia de observar um problema matemático por meio de uma resolução não tradicional com ínfimas abstrações, provocando chances de perceber um evento por outro referencial.
\end{abstract}

Palavras-chave: Paradigma matemático. Ensino e aprendizagem de matemática. Sistemas lineares.

\section{Intuitive notion in the act of teaching and learning mathematics through an activity of teaching linear systems with positive coefficients}

\begin{abstract}
The difficulties in understanding mathematical concepts in basic education are evident in the students' school performance, especially due to the supplanted educational proposals and which fail to bring any interest of the learner closer to the recommended contents. This work aims to address these questions and concerns of researchers in Mathematics Education, establishing three essential assumptions to minimize questions about the teaching and learning of mathematics: Problem, Cause and Paradigm. Methodologically, the proposal is based on offering an intuitive tool for solving 1st degree linear systems with two equations and two unknowns and positive coefficients, through mathematical problems, using a data table. Four mathematical problems will be investigated. It is not enough just to solve the indicated mathematical problem, but to find a way to unveil patterns and establish relationships. It is hoped that this work can assist teachers and students in the teaching and learning process of mathematics, using a strategy of observing a mathematical problem through a nontraditional resolution with tiny abstractions, causing chances of perceiving an event by another reference.
\end{abstract}

Keywords: Mathematical paradigm. Teaching and learning mathematics. Linear systems. 


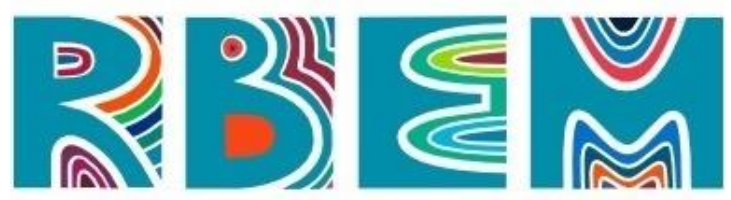

REVISTA BAIANA DE EDUCAÇÃO MATEMÁTICA

\section{Noción intuitiva en el acto de enseñar y aprender matemáticas a través de una actividad de enseñanza de sistemas lineales con coeficientes positivos}

Resumen: Las dificultades en la comprensión de los conceptos matemáticos en la educación básica se evidencian en el desempeño escolar de los estudiantes, especialmente por las propuestas educativas suplantadas y que no logran acercar el interés del educando a los contenidos recomendados. Este trabajo tiene como objetivo abordar estas preguntas e inquietudes de los investigadores en Educación Matemática, estableciendo tres supuestos esenciales para minimizar las preguntas sobre la enseñanza y el aprendizaje de las matemáticas: Problema, Causa y Paradigma. Metodológicamente, la propuesta se basa en ofrecer una herramienta intuitiva para la resolución de sistemas lineales de 1er grado con dos ecuaciones y dos incógnitas y coeficientes positivos, mediante problemas matemáticos, utilizando una tabla de datos. Se investigarán cuatro problemas matemáticos. No basta con resolver el problema matemático indicado, sino encontrar la manera de desvelar patrones y establecer relaciones. Se espera que este trabajo pueda ayudar a profesores y estudiantes en el proceso de enseñanza y aprendizaje de las matemáticas, utilizando una estrategia de observación de un problema matemático a través de una resolución no tradicional con abstracciones minúsculas, provocando posibilidades de percibir un evento por otra referencia.

Palabras clave: Paradigma matemático. Enseñanza y aprendizaje de las matemáticas. Sistemas lineales.

\section{Introdução}

Na contemporaneidade, a sociedade da informação e do conhecimento estabelece que o indivíduo desenvolva novas competências e habilidades, particularmente, na tomada de decisão e na resolução de problemas, de modo que a criatividade passa a ser uma das maiores exigências para a vida e o mercado de trabalho. "Diante de um mundo tecnológico, a matemática vem se tornando uma ferramenta imprescindível em todas as áreas do conhecimento" (DA SILVA et al., 2020, p.152). Seguindo este propósito, percebe-se que a matemática tem um papel efetivo na preparação de indivíduos aptos a afrontar os desafios da modernidade com ousadia e astúcia.

Neste momento é importante esclarecer que entendo matemática como uma estratégia desenvolvida pela espécie humana ao longo de sua história para explicar, para entender, para manejar e conviver com a realidade sensível, perceptível, e com o seu imaginário, naturalmente dentro de um contexto natural e cultural (D’AMBRÓSIO, 2005, p.102).

Segundo Lubachewski e Cerutti (2020) a sociedade está cada vez mais conectada com o mundo tecnológico, vivenciando uma complexidade em que as demandas educacionais exigem do professor, maior articulação e intercâmbio com os fatos contemporâneos, movimento este que abrange a formação inicial e continuada, em que os docentes possam estar em constante aprimoramento. 


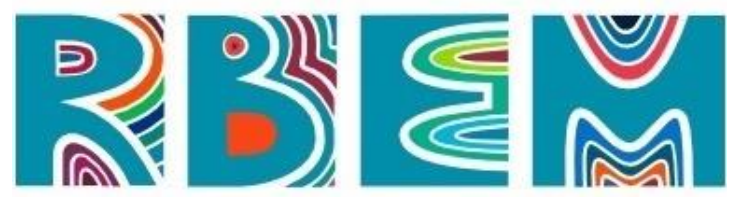

REVISTA BAIANA DE EDUCAÇÃO MATEMÁTICA

Da Costa; Sousa e Cordeiro (2020) afirmam que a discussão do ensino da Matemática na educação básica ocasiona muitas provocações para educadores e pesquisadores, ganhando evidência quando o tema é a necessidade de mudança da prática pedagógica, associada às dificuldades da falta de recursos didáticos e formação continuada de professores para assimilação de metodologias que beneficiem o processo educacional.

Segundo Pontes (2019a), as dificuldades no entendimento de conceitos matemáticos são aparentes no desempenho escolar dos alunos envolvidos, principalmente pelas propostas educacionais superadas e que não impetram aproximar nenhum interesse do aluno pelos conteúdos sugeridos, devido a não existir nenhuma analogia com atividades que satisfaçam às necessidades dos educandos. O processo de ensino e aprendizagem de matemática é plural e constituído de significados, pois reproduzem relações sociais significativas e elementos explicativos sobre as coisas.

Por consequência, nosso trabalho tem como objetivo confrontar essas indagações e inquietudes de estudiosos da Educação Matemática, instituindo três pressupostos imprescindíveis para minimizar questionamentos sobre o ensino e aprendizagem de matemática: Problema, Causa e Paradigma.

A intenção é ressignificar um modelo escolar com responsabilidade pedagógica, alocando o educando como ator de suas aprendizagens, um investigador crítico e conectado com suas realidades e atributos. "Falar em ressignificação é falar sobre mudanças, transitoriedade dos processos do sujeito que aprende, processos de transformação" (SILVA \& MUNIZ, 2020, p.3).

Essa transmutação no processo de ensino e aprendizagem de matemática dar-se-á por meio de eventos associados à resolução de problemas contendo sistemas lineares do $1^{\circ}$ grau, com duas equações e duas incógnitas. A proposta reveste-se em resolver esses eventos por tabelas geradas pela disposição das hipóteses definidas em cada problema recomendado.

\section{O Ensino de Matemática na Educação Básica}

O ensino de matemática nos anos finais do ensino fundamental tem um papel crucial no desenvolvimento cognitivo do aprendiz e, consequentemente, no incremento de técnicas metodológicas que fortaleçam suas habilidades matemáticas. "Parece ponto pacífico 


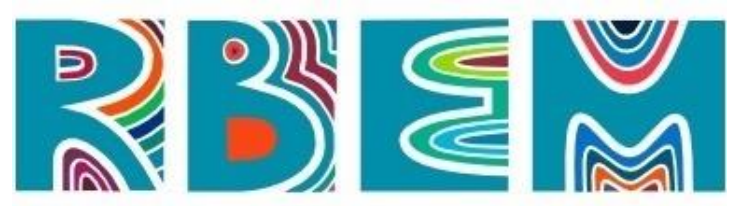

REVISTA BAIANA DE EDUCAÇÃO MATEMÁTICA

considerar que os saberes matemáticos presentes desde os primeiros anos escolares constituem os passos iniciais para a aprendizagem da matemática" (VALENTE, 2016, p.35). Diante de um mundo de avanços científicos e tecnológicos, muitas vezes depararmos com diversos problemas que o ensino de matemática vem afrontando, devido primordialmente pela a falta de integração entre os conteúdos abordados e uma prática direcionada à realidade do educando.

Quando mencionamos aprendizagem matemática, nos referimos ao processo de construção do conhecimento que não tem como objetivo capacitar o estudante para simplesmente repetir o que viu, mas sim, ser capaz de resolver problemas novos (diferente dos já vistos), ou ainda, de construir situações-problema, uma vez que aprender matemática não se resume apenas a resolver problemas (DOS SANTOS; XOTESLEM e GONTIJO, 2020, p.2).

A metodologia empregada pelos educadores que promovem o ensino de matemática nos anos finais do ensino fundamental vem afastando o aprendiz de uma aprendizagem significativa e contextualizada, dificultando todo um processo de entendimento de suas relações, conceitos e aplicações. "A criança ela deve estar apta a desenvolver, intuitivamente, meios de fugir das sequências pré-determinadas e obrigatórias em seu meio escolar" (PONTES, 2019b, p.3). É notório o forte desinteresse dos alunos em aprender matemática, movido por uma extrema dificuldade de percepção dos modelos abstratos com uma prática concreta, retratada nas bancas escolares das escolas brasileiras de educação básica.

Embora o conhecimento matemático seja fruto de um processo, o ensino da matemática está, geralmente, baseado em informações sobre resultados formalizados (definições, procedimentos de cálculos, regras e fórmulas), através das quais são propostas resoluções para problemas e atividades muitas vezes desvinculadas dos significados conceituais que originam a aplicação das práticas propostas (GIRON e DO SACRAMENTO SOARES, 2020, p.3).

Faz-se necessária uma quebra de paradigmas no processo de ensino e aprendizagem de matemática nos anos finais do ensino fundamental, de sorte que a prática tradicional mecânica e fragmentada seja substituída por uma prática ideal interativa e sequenciada. O conhecimento adquirido de forma linear esteja em multiconexão com outros conteúdos. Nossa intenção não é discutir um espaço para competição no ambiente escolar, mas um trabalho em cooperação. 


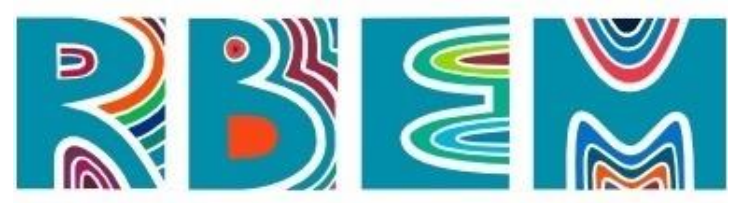

REVISTA BAIANA DE EDUCAÇĀO MATEMÁTICA

$\mathrm{O}$ ato de ensinar e o ato de aprender matemática devem ser contemplados de forma integrada, em conformidade com toda evolução científica e tecnológica do mundo atual.

Diversos conceitos e regras de Matemática desenvolvidos no Ensino Fundamental das séries finais são normalmente trabalhados em sala de aula utilizando uma proposta inadequada e de linguagem formal, fazendo com que a assimilação dos tópicos apresentados seja de difícil compreensão para os envolvidos. No Ensino Fundamental das séries finais, o processo de ensino e aprendizagem de Matemática deve estar respaldado no despertar do jovem aprendiz a utilizar sua criatividade para resolver problemas e tomar decisões acertadas (PONTES, 2018a, p.50).

Pinheiro e Valente (2016) afirmam que ocorra mudança no processo de ensino e aprendizagem de matemática é indispensável desenvolver formas de instruir estudantes nos conteúdos matemáticos, passando a orientar de acordo com as suas perspectivas e necessidades.

Pensar matematicamente envolve tanto dominar as ferramentas matemáticas, seus conceitos e algoritmos, como desenvolver a compreensão de que a matemática é uma ciência com linguagem específica que permite expressar, ler e compreender o mundo, cujos aspectos se relacionam. Pensar matematicamente na relação com o outro, é ser matemático, enquanto que o matemático se ocupa da criação e desenvolvimento de objetos matemáticos (DA SILVA; FEIL, 2020, p.5).

\section{Problema, Causa e Paradigma no ensino de matemática}

Uma das maiores indagações e inquietudes de educadores na educação básica concentram-se sobre a componente curricular de matemática e suas dificuldades no processo ensino e aprendizagem. Dessarte, faz-se imprescindível um realinhamento em todo processo de entendimento da prática pedagógica, desde sua formalização até sua execução, originando alguns questionamentos indispensáveis: De que maneira o professor poderá contribuir para aperfeiçoar seu processo de ensinar matemática? Quais os caminhos que o aluno poderá seguir em busca de melhorar seu aprendizado em matemática? 


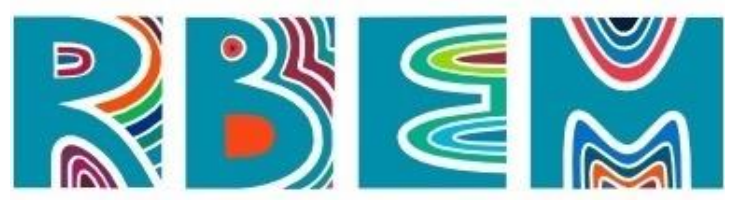

REVISTA BAIANA DE EDUCAÇÃO MATEMÁTICA

Observa-se um protótipo sequencial legítimo, Tabela 1, com palavras-chave visivelmente bem definidas e apropriadas para uma reconstrução de uma proposta viável no processo de ensino e aprendizagem de matemática.

Tabela 1: Problema, Causa e Paradigma no ensino de matemática

\begin{tabular}{ccc}
\hline Problema & Causa & Paradigma \\
\hline $\begin{array}{c}\text { Motivação e interesse do } \\
\text { aluno } \\
\text { Ato de aprender e ensinar }\end{array}$ & Aulas monótonas e chatas & A matemática está em tudo \\
& Falta de contextualização & $\begin{array}{c}\text { Problemas associados à } \\
\text { realidade }\end{array}$ \\
\hline
\end{tabular}

Fonte: elaboração do autor

O problema é fundamentalmente exposto pela falta de motivação e interesse do aluno pela matemática. O facilitador do processo, o professor, precisa alimentar novas possibilidades no ato de ensinar matemática, de maneira que exista uma atração ilimitada pelo ouvinte investigador, o aluno, em seu material explicativo. "O modelo de educação brasileiro atual se mantém inquestionável e previsível, seja na forma de ensinar do professor ou na forma de aprender do aluno" (PONTES et al., 2018b, p.6)

A causa é unicamente associada às aulas monótonas e chatas, sem relações com o cotidiano do aluno nem com outras áreas do conhecimento. É o momento de contextualizar, sem perder o foco nos princípios e relações matemáticas, mesmo do ponto de vista abstrato. "Matemática e Educação são estratégias contextualizadas e interdependentes" (D' AMBRÓSIO, 2005, p.103). A matemática precisa ser tratada por sua essência de representar um modelo que explica as coisas.

O paradigma é a finalização do resultado ideal, definido por eventos que possam gerar desafios aos alunos. A capacidade de memorizar mecanicamente por repetição se caracteriza como um fator escasso frente às novas tecnologias do mundo contemporâneo. A construção de conhecimento matemático deve seguir por um caminho de investigação e resolução de problemas, em sentido antagônico a mera expedição de informação. "Um indivíduo está perante um problema quando se confronta com uma questão a qual não pode dar uma resposta, ou quando não consegue resolver uma situação usando os conhecimentos imediatamente disponíveis" (BUENO et al.,2017, p.13) 


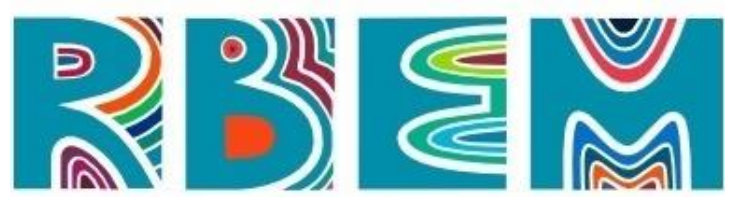

REVISTA BAIANA DE EDUCAÇÃO MATEMÁTICA

Por meio desses pressupostos de ideias, caminhamos para buscar um padrão pedagógico que possa realinhar o processo de ensino e aprendizagem de matemática, aproximando o educando de uma apropriada aprendizagem com significados e integrada a sua realidade. "O ensino de Matemática deve ser proveitoso e ao mesmo tempo benéfico, o indivíduo necessita ter conhecimento sobre o mundo, logo, necessita também, relacionar e estruturar em seu cognitivo as informações que o mundo the transmite" (GIEHL, 2018, p.102).

\section{Exemplos concretos por intermédio dos Sistemas Lineares de Equações de $1^{\circ}$ grau.}

No ensino de Matemática, os sistemas lineares equações de $1^{\circ}$ grau são imprescindíveis para o entendimento de inúmeros problemas aritméticos, além de sua extrema aplicabilidade no dia-a-dia. Resolver um sistema linear de equações do $1^{\circ}$ grau significa encontrar um conjunto solução que satisfaça legitimamente as equações. O intuito de nosso trabalho é oferecer uma ferramenta intuitiva para resolução de sistemas lineares do $1^{\circ}$ grau com duas equações e duas incógnitas por intermédio de problemas matemáticos. Bernard e Cohen (1995) afirmam que a aprendizagem sobre a resolução de equações matemáticas se torna mais expressiva e dinâmica por intermédio da resolução de problemas, diferentemente ao ensino de técnicas improdutivas de significados.

A resolução de problemas é uma das estratégias de ensino e aprendizagem da Matemática que mais tem favorecido o desenvolvimento de capacidades no aluno em pensar produtivamente, quando aplicada de maneira significativa. Enquanto metodologia de ensino-aprendizagem busca oferecer ao professor uma forma de ensinar Matemática através da construção de conhecimentos. Enquanto estratégia para o aluno promove o pensar matemático e o traz para dentro do contexto de aprendizagem, de modo a torná-lo não apenas um aprendiz, mas um agente produtor de conhecimento, pois ao resolver problema poderá externar seu saber e ser desafiado à descoberta de novos saberes (PINHEIRO \& MEDEIROS, 2020, p.6-7).

No propósito de gerar desafios que possam instigar os educandos a habituar-se plenamente com a matemática, nossa proposta é resignificar o modo de operacionalizar a deliberação da solução de um problema matemático, de maneira que o aluno empregue toda sua intuição para resolvê-lo. "Um rápido olhar sobre o desenvolvimento do conhecimento 


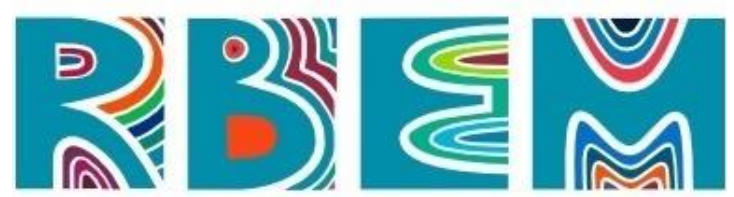

REVISTA BAIANA DE EDUCAÇÃO MATEMÁTICA

matemático, ao longo do tempo, nos leva a perceber que a atividade de resolução de problemas lhe serve de motor" (DE MEDEIROS, 2001, p.2). Faz-se indispensável criar eventos que permaneçam associados intrinsecamente a realidade do aluno, admitindo um olhar diferente sobre o desafio proposto.

\begin{abstract}
Em linhas gerais, o ensino de matemática tem sido tema de muitas reflexões, levando em consideração o grande avanço da tecnologia e as constantes mudanças culturais e profissionais frente às diferentes metodologias de ensino. Faz-se necessário, no entanto, adequar as práticas pedagógicas com a realidade dos alunos, buscando atingir os objetivos do processo de ensino e aprendizagem e proporcionar situações em que os alunos realmente aprendam, compreendendo os conhecimentos da disciplina como parte integrante do cotidiano, não somente nos conteúdos ministrados no ambiente escolar (SILVA et al., 2020, p.6).
\end{abstract}

A metodologia de resolução tradicional de um problema matemático mecânico precisa ser substituída por métodos intuitivos, criativos e de raciocínio lógico, de tal forma que o aluno esteja motivado e pronto para desenvolver seus conhecimentos e saberes. Desta forma, apresentaremos nesta seção quatro eventos matemáticos abordados no estudo de sistemas lineares de equações do tipo: $\left\{\begin{array}{c}x+y=p \\ m x+n y=q\end{array}\right.$, onde $\mathrm{m}, \mathrm{n}, \mathrm{p}$ e q são números naturais. Por ser

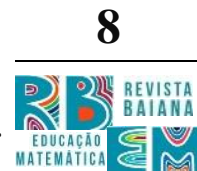
uma noção intuitiva para o entendimento de sistemas lineares do tipo $2 \times 2$ com coeficientes positivos. Os eventos são propostas do autor para uma melhor aprendizagem do conteúdo e que podem ser empregadas em turmas dos anos finais do ensino fundamental.

A ideia é expor o modelo matemático do problema sugerido, todavia resolvê-lo a partir de uma tabela de prováveis resultados esperados, que denotaremos por protótipo matemático. Essa tabela é composta de quatro colunas: $1^{\circ}$ da incógnita $X, 2^{\circ}$ da incógnita $Y, 3^{\circ}$ da hipótese um, gerada pela soma das incógnitas e $4^{\circ}$ da hipótese dois, definida por uma equação linear composta das incógnitas X e Y.

\title{
Evento Um:
}

Um colegial pagou um passeio da escola de 7 reais em cédulas de um real e dois reais. Sabe-se que, para este pagamento, o colegial utilizou 5 cédulas. Determine as quantidades de cédulas de um real e dois reais que foram utilizadas no pagamento do passeio. 


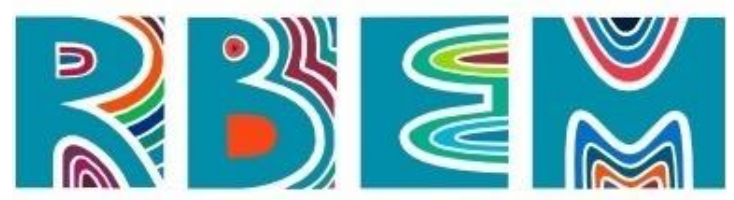

Modelo matemático: $\left\{\begin{array}{c}x+y=5 \\ x+2 y=7\end{array}\right.$

Protótipo matemático:

\begin{tabular}{cccc}
\hline $\mathrm{X}$ & $\mathrm{Y}$ & Hipótese 1 & Hipótese 2 \\
$\mathrm{X}$ & $\mathrm{X}+\mathrm{Y}$ & $\mathrm{X}$ \\
$\mathrm{R} \$ 1$ & $\mathbf{4}$ & $\mathbf{5}$ & $\mathbf{1 + 2 . 4 = 9}$ \\
\hline 1 & $\mathbf{3}$ & $\mathbf{5}$ & $\mathbf{2 + 2 . 3 = 8}$ \\
2 & $\mathbf{2}$ & $\mathbf{5}$ & $\mathbf{3}+\mathbf{2 . 2}=\mathbf{7}$ \\
4 & $\mathbf{1}$ & $\mathbf{5}$ & $\mathbf{4 + 2 . 1}=\mathbf{6}$ \\
\hline
\end{tabular}

\section{Evento Dois:}

Ana é a irmã mais velha de João. A soma das idades de Ana e João é 12 e a diferença é 8. Descubra a idade de Ana e João.

Modelo matemático: $\left\{\begin{array}{c}x+y=12 \\ x-y=8\end{array}\right.$

Protótipo matemático:

\begin{tabular}{cccc}
\hline $\mathbf{X}$ & $\mathbf{Y}$ & Hipótese 1 & Hipótese 2 \\
Ana & João & X+Y & X-Y \\
\hline 7 & 5 & 12 & $7-5=2$ \\
8 & 4 & 12 & $8-4=4$ \\
9 & 3 & 12 & $9-3=6$ \\
10 & 2 & 12 & $10-2=8$ \\
11 & 1 & 12 & $11-1=10$ \\
\hline
\end{tabular}

\section{Evento Três:}

Em uma praça da cidade há 10 crianças andando de bicicleta ou de skate. No total, há 26 rodas girando pela praça. Quantas crianças andam de bicicleta e quantas andam de skate?

Modelo matemático: $\left\{\begin{array}{c}x+y=10 \\ 4 x+2 y=26\end{array}\right.$ 


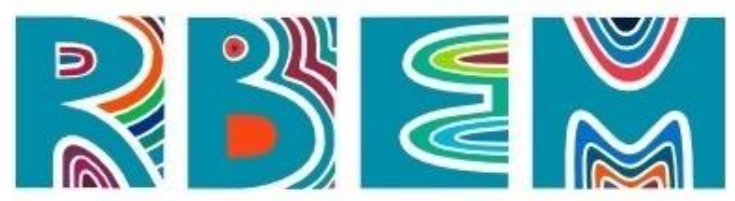

Protótipo matemático:

\begin{tabular}{cccc}
\hline X & Y & Hipótese 1 & Hipótese 2 \\
Ana & João & X+Y & 4 X+2Y \\
\hline 1 & 9 & 10 & $4.1+2.9=22$ \\
2 & 8 & 10 & $4.2+2.8=24$ \\
3 & 7 & 10 & $4.3+2.7=26$ \\
4 & 6 & 10 & $4.4+2.6=28$ \\
5 & 5 & 10 & $4.5+2.5=30$ \\
6 & 4 & 10 & $4.6+2.4=32$ \\
7 & 3 & 10 & $4.7+2.3=34$ \\
9 & 2 & 10 & $4.8+2.2=36$ \\
\hline
\end{tabular}

\section{Evento quatro:}

Um professor de matemática passa uma atividade para seus alunos. José foi convidado a resolver toda atividade no quadro, de forma que cada exercício resolvido corretamente ele ganharia 3 pontos e cada exercício errado perderia 2 pontos. Ao final da atividade, José dos 6 exercícios respondidos, conseguiu acumular 8 pontos. Quantos exercícios José acertou e quantos ele errou?

Modelo matemático: $\left\{\begin{array}{c}x+y=6 \\ 3 x-2 y=8\end{array}\right.$

Protótipo matemático:

\begin{tabular}{cccc}
\hline $\mathbf{X}$ & $\mathbf{Y}$ & Hipótese 1 & Hipótese 2 \\
$\begin{array}{c}\text { Exercício } \\
\text { Certo }\end{array}$ & $\begin{array}{c}\text { Exercício } \\
\text { Errado }\end{array}$ & $\mathbf{X}+\mathbf{Y}$ & $\mathbf{3 X - 2 Y}$ \\
\hline $\mathbf{0}$ & 6 & 6 & $3.0-2.6=-12$ \\
$\mathbf{1}$ & $\mathbf{5}$ & $\mathbf{6}$ & $3.1-2.5=-7$ \\
$\mathbf{2}$ & $\mathbf{4}$ & $\mathbf{6}$ & $3.2-2.4=-2$ \\
$\mathbf{3}$ & $\mathbf{3}$ & $\mathbf{6}$ & $3.3-2.3=3$ \\
$\mathbf{4}$ & $\mathbf{2}$ & $\mathbf{6}$ & $3.4-2.2=8$ \\
$\mathbf{5}$ & $\mathbf{1}$ & $\mathbf{6}$ & $3.5-2.1=13$
\end{tabular}




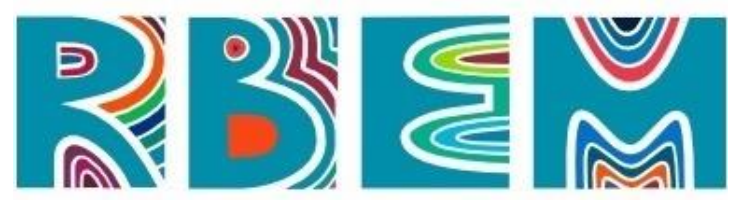

REVISTA BAIANA DE EDUCAÇÃO MATEMÁtica

0

6

$3.6-2.0=16$

Nota-se, que os eventos seguem um raciocínio bastante peculiar, pois a partir da representação das incógnitas $\mathrm{X}$ e Y. Toma-se a soma $x+y=p$, como hipótese um. A seguir define $m x+n y=q$, como hipótese dois. Pelas tabelas apresentadas, as soluções encontradas dos eventos um, dois, três e quatro são, respectivamente, $(3,2),(10,2)$ e $(3,7)$ e $(4,2)$.

É possível que o aluno, inicialmente, não tenha plena consciência da estratégia que deva seguir, mas entrará em convergência a partir do momento que os eventos forem construídos e que os modelos matemáticos façam sentido em sua percepção intuitiva.

Não basta apenas resolver o problema matemático proposto, contudo encontrar um caminho para desvendar padrões e constituir relações, fundamentalmente a ideia de se trabalhar com o processo de ensino e aprendizagem de Matemática por meio da resolução de problemas tem como base permitir que os educandos compreendam inteiramente os métodos matemáticos, sempre acompanhados pela a orientação do professor.

A educação matemática não pode se limitar à transmissão de conhecimentos e modelos pré-estabelecidos, percebe-se que qualquer aprendizagem é um processo cognitivo lento, requerendo do aluno estímulos necessários para prosseguir investigando, sujeitando a atividades que certifiquem o desenvolvimento de suas habilidades e seu sentimento de competência pela etapa concretizada. Para Cai e Lester (2012, p.156),“os professores devem aceitar que as habilidades dos alunos em resolver problemas frequentemente se desenvolvem lentamente, exigindo, assim, uma atenção assistida, em longo prazo".

Não podemos continuar num universo ramificado, de erros e acertos, da passividade e investigação, apresentando de um lado o professor e do outro o educando, se faz necessário uma parceria entre ambos, de modo à descoberta natural de novos caminhos para a produção do conhecimento e saber. "O ato de aprender matemática do aluno requer criatividade, curiosidade e desejo por novos conhecimentos e saberes. $\mathrm{O}$ ato de ensinar matemática do professor é um fator prioritário para a compreensão concreta dos modelos matemáticos abstratos" (PONTES, 2018c, p.120).

\section{Considerações Finais}




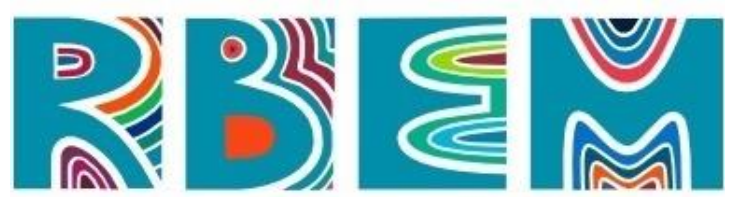

REVISTA BAIANA DE EDUCAÇÃO MATEMÁTICA

O protótipo matemático sugerido é um exercício de realinhamento das estruturas básicas no intuito de fortalecer o pensamento matemático, por esta razão é imperativo que o ato de ensinar do professor e o ato de aprender do aluno permaneçam inteiramente integrados na intenção da assimilação de uma real transformação no ensino e aprendizagem de matemática.

Com base nos elementos exibidos, a proposta em ajustar uma ferramenta intuitiva para resolução de sistemas lineares do $1^{\circ}$ grau com duas equações, duas incógnitas e coeficiente positivos, pode facilmente cooperar para aperfeiçoar a aprendizagem e o desempenho cognitivo dos alunos, assim como, novas contribuições metodológicas ao professor para o ensino de matemática.

O emprego de problemas matemáticos na compreensão de conceitos e princípios é fundamental para o entendimento da abstração matemática, e, consequentemente, gerando maior motivação entre as partes interessadas: professor e aluno. Nota-se que a intenção da proposta não foi de resolver os eventos pelos métodos e técnicas conhecidas, mas permitir que o aluno desenvolva toda sua habilidade intuitiva na ação de encontrar a solução ideal, sempre auxiliado pelo professor.

Dessa forma, reconhecer as percepções sobre o pensamento matemático e a ressignificação do método de ensino e aprendizagem de matemática se compõem um passo importante para uma adequada transformação nas bancas escolares, de modo que alunos e professores estejam conectados e possam constituir a verdadeira base educacional qualificada e motivada em busca do saber.

Assim sendo, os questionamentos recomendados são respondidos, a partir das reflexões sobre a importância do professor e do aluno no aperfeiçoamento de todo processo de ensino e aprendizagem de matemática, originando caminhos para o desenvolvimento do pensamento matemático, de maneira a fortalecer a prática educacional.

Diante do exposto, que este trabalho, possa auxiliar professores e alunos no processo de ensino e aprendizagem de matemática, empregando uma estratégia de observar um evento matemático por intermédio de uma resolução não tradicional com mínimas abstrações, bem como fazê-los compreender que gerar oportunidades de perceber um problema, por outro referencial, pode ser algo bem-sucedido na construção do conhecimento. Espera-se que 


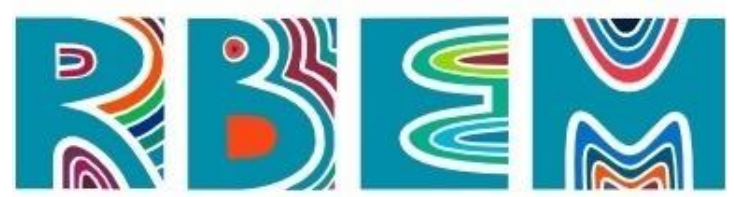

REVISTA BAIANA DE EDUCAÇÃO MATEMÁTICA

pesquisadores e educadores possam contribuir em trabalhos futuros, apresentando outros desdobramentos no processo de ensino e aprendizagem de matemática na educação básica.

\section{Referências}

BERNARD, J.; COHEN, M. Uma integração dos métodos de resolução de equações numa sequência evolutiva de aprendizado. In: COXFORD, A.; SHULTE, A. (Org). As ideias da álgebra. Tradução de Hygino Domingues. São Paulo: Atual, 1995. cap. 10, p. 111-126.

BUENO, Simone; ALENCAR, Edvonete; MILLONES, Teresa. Reflexões e desafios da resolução de problemas nas aulas de Matemática: um ensaio teórico. Educação Matemática Debate, v. 1, n. 1, p. 9-27, 2017.

CAI, Jinfa; LESTER, Frank. Por que o ensino com resolução de problemas é importante para a aprendizagem do aluno. Boletim Gepem, v. 60, p. 147-162, 2012.

DA COSTA, Renato Pinheiro; SOUSA, Camila; CORDEIRO, Leonardo Zenha. O ensino de Matemática na Base Nacional Comum Curricular nos anos finais do Ensino Fundamental. Ensino em Re-Vista, p. 572-594, 2020.

DA SILVA, Robespierre Cocker Gomes et al. O Ato de Ensinar e o Ato de Aprender Matemática na Ótica do Professor Edel Alexandre Silva Pontes. Revista Psicologia \& Saberes, v. 9, n. 16, p. 151-162, 2020.

DA SILVA, Fernando Siqueira; FEIL, Gabriel Sausen. A possibilidade da constituição do ser matemático no agir comunicativo. Revista Eletrônica de Educação Matemática, v. 15, n. 2, p. $1-18,2020$.

D AMBRÓSIO, Ubiratan. Sociedade, cultura, matemática e seu ensino. Educação e pesquisa, v. 31, n. 1, p. 99-120, 2005.

DOS SANTOS, Cleiton Rodrigues; XOTESLEM, Wesley Vieira; GONTIJO, Cleyton Hercules. AS IMPLICAÇÕES DO USO DE RECURSOS TECNOLÓGICOS NO PROCESSO DE APRENDIZAGEM/ENSINO DE GEOMETRIA PLANA. VIII Jornada Nacional de Educação Matemática. Universidade de Passo Fundo. 2020.

GIEHL, Leidi Katia. A Aprendizagem Significativa no Ensino da Matemática: Discussões e Experiências. Revista Pleiade, v. 12, n. 26, p. 99-107, 2018.

GIRON, Graziela Rossetto; DO SACRAMENTO SOARES, Eliana Maria. Nicho de Aprendizagem Matemática: uma experiência de ensinar e aprender matemática em convivência. Horizontes, v. 38, n. 1, p. e020053-e020053, 2020. 


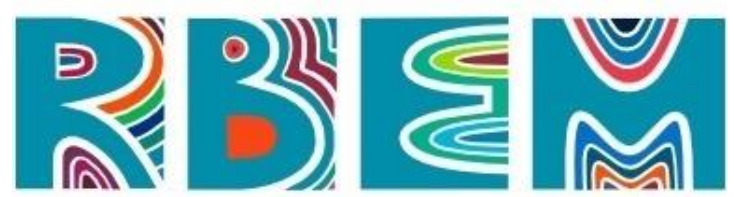

REVISTA BAIANA DE EDUCAÇÃO MATEMÁTICA

LUBACHEWSKI, Gesseca Camara; CERUTTI, Elisabete. Metodologias ativas no ensino da matemática no anos iniciais: aprendizagem por meio de jogos. RIDPHE_R Revista Iberoamericana do Patrimônio Histórico-Educativo, v. 6, p. e020018-e020018, 2020.

DE MEDEIROS, Kátia Maria. O contrato didático e a resolução de problemas matemáticos em sala de aula. 2001.

PINHEIRO, N. V. L.; VALENTE, W. R. Chega de decorar a tabuada! As Cartas de Parker e a árvore do cálculo na ruptura de uma tradição. Educação Matemática em Revista. SBEM-RS, ano, v. 16, p. 16-37, 2015.

PINHEIRO, J. M. DE Q.; MEDEIROS, K. M. DE. As perguntas para desenvolver estratégias: Álgebra e Resolução de Problemas no Ensino Médio. Revista Baiana de Educação Matemática, v. 1, p. e202010, 23 out. 2020.

PONTES, Edel Alexandre Silva. Modelo de ensino e aprendizagem de matemática baseado em resolução de problemas através de uma situação-problema. Revista Sítio Novo, v. 2, n. 2, p. 44-56, 2018.

PONTES, Edel Alexandre Silva et al. Abordagens Imprescindíveis no Ensino Contextualizado de Matemática nas Séries Iniciais da Educação Básica. RACE-Revista de Administração do Cesmac, v. 1, p. 3-15, 2018.

PONTES, Edel Alexandre Silva. O professor ensina e o aluno aprende: questões teóricas no processo de ensino e aprendizagem de Matemática. RACE-Revista de Administração do do Cesmac, v. 4, p. 111-124, 2019.

PONTES, Edel Alexandre Silva. Os Quatro Pilares Educacionais no Processo de Ensino e Aprendizagem de Matemática. Revista Iberoamericana de Tecnología en Educación y Educación en Tecnología, n. 24, p. e02-e02, 2019.

PONTES, Edel Alexandre Silva. Método de polya para resolução de problemas matemáticos: uma proposta metodológica para o ensino e aprendizagem de matemática na educação básica. HOLOS, v. 3, p. 1-9, 2019.

SILVA, Ana Gisnayane Sousa; DE SOUSA, Francisco Jucivânio Félix; DE MEDEIROS, Jarles Lopes. O ensino da matemática: aspectos históricos. Research, Society and Development, v. 9, n. 8, p. e488985850-e488985850, 2020.

SILVA, G. C.; MUNIZ, C. A. Ressignificação da Matemática por estudantes de Pedagogia: jogar e reaprender para ensinar. Revista Baiana de Educação Matemática, v. 1, p. e202001, 22 jul. 2020.

VALENTE, Wagner Rodrigues. A matemática nos primeiros anos escolares: elementos ou rudimentos? História da Educação, v. 20, n. 49, p. 33-47, 2016. 


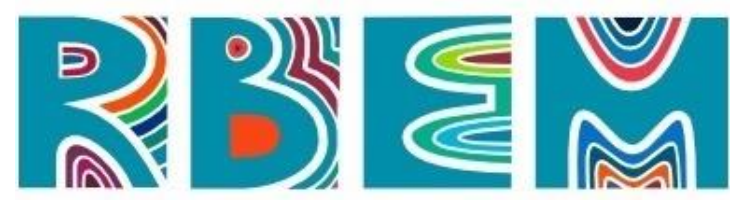

REVISTA BAIANA DE EDUCAÇÃO MATEMÁTICA

Artigo submetido em: 24/03/2021

Artigo aceito em: 26/05/2021 